\title{
Periodicity During Hypercapnic and Hypoxic Stimulus Is Crucial in Distinct Aspects of Phrenic Nerve Plasticity
}

\author{
I. STIPICA ${ }^{1}$, I. PAVLINAC DODIG ${ }^{1}$, R. PECOTIC ${ }^{1}$, Z. DOGAS $^{1}$, Z. VALIC $^{2}$, M. VALIC $^{1}$ \\ ${ }^{1}$ Department of Neuroscience, University of Split School of Medicine, Split, Croatia, ${ }^{2}$ Department \\ of Physiology, University of Split School of Medicine, Split, Croatia
}

Received February 12, 2015

Accepted July 10, 2015

On-line November 24, 2015

\section{Summary}

This study was undertaken to determine pattern sensitivity of phrenic nerve plasticity in respect to different respiratory challenges. We compared long-term effects of intermittent and continuous hypercapnic and hypoxic stimuli, and combined intermittent hypercapnia and hypoxia on phrenic nerve plasticity. Adult, male, urethane-anesthetized, vagotomized, paralyzed, mechanically ventilated Sprague-Dawley rats were exposed to: acute intermittent hypercapnia (AIHc or $\mathrm{AIHC}_{02}$ ), acute intermittent hypoxia $(\mathrm{AIH})$, combined intermittent hypercapnia and hypoxia $(\mathrm{AIHCH})$, continuous hypercapnia $(\mathrm{CHc})$, or continuous hypoxia $(\mathrm{CH})$. Peak phrenic nerve activity (pPNA) and burst frequency were analyzed during baseline (TO), hypercapnia or hypoxia exposures, at 15,30 , and $60 \mathrm{~min}(\mathrm{~T} 60)$ after the end of the stimulus. Exposure to acute intermittent hypercapnia elicited decrease of phrenic nerve frequency from $44.25 \pm 4.06$ at T0 to $35.29 \pm 5.21$ at $\mathrm{T} 60,(P=0.038, A I H c)$ and from $45.5 \pm 2.62$ to $37.17 \pm 3.68$ breaths $/ \mathrm{min}\left(\mathrm{P}=0.049, \mathrm{AIHc}_{\mathrm{O} 2}\right)$, i.e. frequency phrenic long term depression was induced. Exposure to $\mathrm{AIH}$ elicited increase of pPNA at T60 by $141.0 \pm 28.2 \%$ compared to baseline $(P=0.015)$, i.e. phrenic long-term facilitation was induced. Exposure to $\mathrm{AIHcH}, \mathrm{CHc}$, or $\mathrm{CH}$ protocols failed to induce long-term plasticity of the phrenic nerve. Thus, we conclude that intermittency of the hypercapnic or hypoxic stimuli is needed to evoke phrenic nerve plasticity.

\section{Key words}

Hypercapnia • Hypoxia • Phrenic nerve • Respiratory plasticity • Rat

\section{Corresponding author}

M. Valic, University of Split School of Medicine, Department of Neuroscience, Soltanska 2, 21000 Split, Croatia. E-mail: maja.valic@mefst.hr

\section{Introduction}

Neuroplasticity is an important property of the respiratory system whose functionality and biological significance has been recognized in recent years. Its clinical significance is related to the pathology of several medical conditions (Dale et al. 2014), including sleeprelated breathing disorders characterized by intermittent hypoxic episodes through the night. The most frequently studied model of respiratory plasticity is respiratory longterm facilitation (LTF) following acute intermittent hypoxia (Mitchell et al. 2001, Feldman et al. 2003). Its conceptual development and possible mechanisms have been in the research focus for years, leading to conclusions regarding the modulation of the phrenic LTF (pLTF) (Bach and Mitchell 1996, Valic et al. 2010). However, a variety of factors such as periodicity versus continuity, intensity, duration and frequency of hypoxic episodes, and background oxygen level are shown to play an important role in the development of pLTF (Mateika and Sandhu 2011, Devinney et al. 2013, Dick et al. 2004, 2007, Bach and Mitchell 1998). Studies have revealed that brief episodes of simulated apnea (Mahamed and Mitchell 2008), and intermittent negative pressure applied to the upper airway (Ryan and Nolan 2009) can produce respiratory LTF. Furthermore, it has been shown that only periodic but not continuous hypoxic stimulus can 
produce respiratory LTF (Baker and Mitchell 2000), showing that intermittency might be the most important factor for inducing LTF.

Whilst the consequences and benefits of intermittent hypoxic exposures have been extensively studied, the effects of repetitive hypercapnia have not been well documented despite the fact that it can also alter respiratory neural output. Contrasting results from previous studies reveal that episodic intracarotid hypercapnia induced pLTF (Morris et al. 1996), while systemic hypercapnia, which stimulated both central and peripheral chemoreceptors, caused respiratory long-term depression (LTD) (Bach and Mitchell 1998). Furthermore, it has been shown that severe intermittent hypercapnia $\left(10 \% \mathrm{CO}_{2}\right)$ evoked LTD of the phrenic nerve activity ( $\mathrm{pLTD}$ ) in some experimental designs (Bach and Mitchell 1998), but failed to do so in similar studies (Baker et al. 2001) pointing to continuous stimulus as an adequate one to evoke pLTD (Kinkead et al. 2001). The reasons underlying this discrepancy are unknown but one possibility might be rat substrain differences. Furthermore, pattern dependence of LTD seen in previous studies might be partially explained by the activation of distinctive neurotransmitter systems. There is sufficient evidence to suggest that the respiratory control system is a complex neural circuit that integrates serotonergic and noradrenergic systems that interact in a "push-pull" manner in modulation of respiratory plasticity (Kinkead et al. 2001, Baker et al. 2001).

Respiratory plasticity following acute intermittent hypoxia is primarily expressed as a long lasting increase of phrenic nerve amplitude, whereas changes of the phrenic nerve burst frequency appear to be varying. While amplitude LTF might be the result of modulation of respiratory motor neurons (Fuller et al. 2000, Baker-Herman and Mitchell 2002, 2008), frequency LTF is most likely the result of brainstem respiratory rhythm generating neurons modulation (Baker-Herman and Mitchell 2008). On the other hand, opposing effects of episodic and continuous hypercapnia result in transient depression of the phrenic burst frequency (Bach and Mitchell 1998, Baker et al. 2001).

Previous studies of intermittent hypercapnia or hypoxia pointed to a puzzling feature of the respiratory control system (Harris et al. 2006, Mateika and Sandhu 2011, Devinney et al. 2013), indicating that altering the pattern of the stimulus might have an impact on the expression and magnitude of respiratory plasticity
(Mateika and Sandhu 2011). The majority of previous LTF studies have utilized acute intermittent isocapnic hypoxia as an inducing stimulus (Mitchell et al. 2001), but there is no published study demonstrating the effect of concomitant hypoxia and hypercapnia. Therefore, this study was undertaken to determine pattern sensitivity of phrenic nerve plasticity in respect to different respiratory challenges bringing them together into a single study on the same substrain of Sprague-Dawley rats, making the direct comparison more meaningful. We compared the long-term effects of intermittent and continuous hypercapnic and hypoxic stimuli on phrenic nerve plasticity. In addition, the novelty of this study was to investigate the impact of combined intermittent hypoxia and hypercapnia on phrenic nerve plasticity.

\section{Material and Methods}

The protocol for this study was approved by the Ethical Committee for Biomedical Research of the University of Split School of Medicine, Split, Croatia. All experiments were done in accordance with National Research Council's guide for the care and use of laboratory animals.

\section{Experimental preparation}

Experiments were conducted on 46 adult male Sprague-Dawley (Harlan, Italy) rats weighing 280-330 g (Animal facility of the University of Split School of Medicine). Anesthesia of a $20 \%$ solution of urethane in $0.9 \%$ saline $(1.2 \mathrm{~g} / \mathrm{kg}$; supplemental dose $0.2 \mathrm{~g} / \mathrm{kg})$ was injected intraperitoneally. The adequacy of anesthesia was assessed by the absence of withdrawal reflex after noxious paw pinch.

The femoral artery and vein were cannulated bilaterally for blood pressure monitoring, blood sampling and drug injection. Blood samples for the determination of blood gases and $\mathrm{pH}$ (RapidLab 348, Bayer Diagnostics, Sudbury, UK) were taken at the regular intervals and arterial blood gases and $\mathrm{pH}$ were maintained within physiological limits by the adjustment of ventilation or infusion of bicarbonates, if needed. All animals were vagotomized bilaterally to eliminate pulmonary stretch receptors input. Rectal temperature was monitored by digital thermometer and maintained between 37 and $38.5^{\circ} \mathrm{C}$.

The trachea was cannulated through a midline ventral neck incision. The animals were artificially 
ventilated through a tracheostomy using a respirator for small animals (SAR 830-P, CWE, Ardmore, USA). The initial parameters of artificial ventilation were a respiratory rate of 50 breaths/min, an inspiratory pressure of $12 \mathrm{~cm} \mathrm{H}_{2} \mathrm{O}$, an inspiratory flow range $250-350 \mathrm{ml} / \mathrm{min}$, and a positive end-expiratory pressure of $2-3 \mathrm{~cm} \mathrm{H} \mathrm{H}_{2} \mathrm{O}$, and later they were adapted according to blood gases and capnography. The concentration of $\mathrm{CO}_{2}$ was continuously monitored with a Gemini respiratory gas analyzer (CWE inc., Ardmore, USA) and adjusted by changing the respiratory rate and/or inspiratory time. The animals were paralyzed with the neuromuscular blocking agent rocuronium bromide (Organon, $50 \mathrm{mg}$ in $5 \mathrm{ml}$, $1.33 \mathrm{mg} / \mathrm{kg})$.

The rats were placed in a prone position in a stereotaxic instrument (Lab Standard, Stoelting, USA). The right phrenic nerve was dissected using the dorsal approach at the level of the $\mathrm{C} 5$ nerve rootlet, mounted on bipolar silver wire electrodes and covered with silicone gel to prevent from drying.

\section{Experimental groups}

Following surgical preparation, there were six experimental groups. Three groups were exposed to hypercapnia (15\% $\mathrm{CO}_{2}$ in air), two to hypoxia $\left(9 \% \mathrm{O}_{2}\right.$ in $\mathrm{N}_{2}$ ), and one to combined hypercapnia and hypoxia $\left(15 \% \mathrm{CO}_{2}\right.$ and $9 \% \mathrm{O}_{2}$ in $\mathrm{N}_{2}$ ).

\section{Hypercapnic groups}

Two groups of animals were exposed to five 3-min long episodes of intermittent hypercapnia, separated by 3-min recovery periods when the animals were ventilated either with room air (group AIHc, $n=8$ ), or with $50 \%$ oxygen in $\mathrm{N}_{2}$ (group $\mathrm{AIHc}_{\mathrm{O} 2}, \mathrm{n}=6$ ) to compare the impact of background oxygen level on hypercapnic responses. One group of animals was exposed to continuous hypercapnia for a duration of $30 \mathrm{~min}$ (group $\mathrm{CHc}, \mathrm{n}=6$ ). Prior to and following hypercapnic stimulation, $\mathrm{CHc}$ group was ventilated with room air.

\section{Hypoxic groups}

One group was exposed to five 3-min long episodes of acute intermittent hypoxia, separated by 3 -min recovery periods when the animals were ventilated with a 50/50 oxygen/nitrogen mixture (group AIH, $n=7$ ). One group was exposed to 30 min of continuous hypoxia (group $\mathrm{CH}, \mathrm{n}=7$ ). Prior to and following hypoxic stimulation, both groups, $\mathrm{AIH}$ and $\mathrm{CH}$ were ventilated with a 50/50 oxygen/nitrogen mixture.

\section{Combined hypercapnia and hypoxia group}

One group of animals (group $\mathrm{AIHcH}, \mathrm{n}=8$ ) was exposed to combined intermittent hypercapnia and hypoxia in the same manner (5 exposures each lasting $3 \mathrm{~min}$, separated by a $3-\mathrm{min}$ recovery period). Prior to and following stimulation, this group was ventilated with a 50/50 oxygen/nitrogen mixture.

To examine the effects of the anesthetic urethane and/or other time dependent factors on the viability and stability of preparation, experiments were conducted on an additional 5 rats (time-control) with the exception that these animals were not exposed to hypercapnia or hypoxia. Throughout the duration of the experiment there were no significant changes in the phrenic nerve activity, indicating the stability of the preparation (Carev et al. 2010, Pavlinac Dodig et al. 2012, Ivancev et al. 2013, Marinov et al. 2013).

\section{Protocols}

Following the completion of surgical procedures, a minimum of $30 \mathrm{~min}$ was allowed for the stabilization of phrenic nerve activity in baseline conditions. Baseline phrenic nerve activity was achieved by manipulating ventilator parameters while monitoring $\mathrm{ET}_{\mathrm{CO} 2}$ levels. The apneic threshold was determined and the initial $\mathrm{PET}_{\mathrm{CO} 2}$ was set about 3-4 mm $\mathrm{Hg}$ above this level. The control blood sample was taken prior to hypercapnic or hypoxic stimulus. During hypercapnia or hypoxia protocol blood sampling was avoided in order to minimize the overall blood volume loss from the animals. Arterial blood samples were taken at three posthypercapnic or post-hypoxic periods $(15,30$ and $60 \mathrm{~min}$ following the hypercapnic or hypoxic stimulus).

At the end of all the experiments, rats were euthanized with an overdose of urethane followed by an intravenous injection of potassium chloride.

\section{Data acquisition and analysis}

Phrenic nerve activity was amplified, filtered (band-pass $300 \mathrm{~Hz}-10 \mathrm{kHz}$ ) and rectified. The movingtime average of phrenic nerve activity was obtained using the MA-1000 Moving Averager, System 1000 Modulator instrumentation (CWE Inc., Ardmore, USA) with a $50 \mathrm{~ms}$ time constant. The processed signal- phrenic neurogram (PNG) was recorded using the PowerLab system 
(ADInstruments, Castle Hill, Australia) and analyzed by PowerLab software, Chart for Windows version 5.4.2 and Scope for Windows version 3.8 (ADI Instruments, Castle Hill, Australia). There were nine time points for data analysis: before the first hypercapnia/hypoxia (T0), during hypercapnic/hypoxic episodes 1-5 (TH1-TH5), and 15 (T15), 30 (T30) and 60 (T60) min after the last hypercapnic/hypoxic stimulation. From the digital record of the phrenic nerve activity, we analyzed peak phrenic nerve activity (pPNA), phrenic burst frequency (f), inspiratory duration (Ti), expiratory duration (Te) and respiratory cycle total duration (Ttot). The phrenic nerve activity was averaged once it had reached the steadystate, and a minimum of a $20 \mathrm{~s}$ signal was required for successful analysis. Average amplitude data were normalized as the percentage change from baseline activity (immediately before the first hypercapnia or hypoxia).

Data were analyzed using MedCalc (Ostend, Belgium). A one- and two-way repeated measures ANOVA (within and between groups) was performed followed by Bonferroni post-hoc correction to provide information for all pair wise comparisons of interest within groups at the same time point during the protocol. Data were presented as mean \pm SEM. A P-value of 0.05 was considered statistically significant.

\section{Results}

\section{Effects of hypercapnic stimuli}

Acute intermittent hypercapnia evoked depression of the respiratory frequency $60 \mathrm{~min}$ after the end of the last hypercapnic stimulus, i.e. respiratory frequency decreased from $44.25 \pm 4.06$ breaths $/ \mathrm{min}$ at $\mathrm{T} 0$ to $35.29 \pm 5.21$ breaths $/ \mathrm{min}$ at $\mathrm{T} 60$ in the AIHc group $(\mathrm{P}=0.038$; Figs 1 and 2$)$, and from $45.5 \pm 2.62$ breaths $/ \mathrm{min}$ at $\mathrm{T} 0$ to $37.17 \pm 3.68$ breaths $/ \mathrm{min}$ at $\mathrm{T} 60$ in the $\mathrm{AIHc}_{\mathrm{O} 2}$ group $(\mathrm{P}=0.049)$, showing that frequency $\mathrm{pLTD}$ was developed. However, exposure to acute intermittent hypercapnia protocol did not evoke persistent longlasting changes of the pPNA $60 \mathrm{~min}$ after the end of the stimuli $(-4.8 \pm 21.6 \%$ in the AIHc group, $\mathrm{P}=1$; Figs 1 and 3 , and $-22.3 \pm 11.6 \%$ in the $\mathrm{AIHc}_{\mathrm{O} 2}$ group, $\mathrm{P}=0.67$ ).

Continuous hypercapnia failed to evoke frequency pLTD $60 \mathrm{~min}$ after the end of the stimulus, i.e. respiratory frequency was $40.29 \pm 2.69$ breaths $/ \mathrm{min}$ at T0, and $38.14 \pm 2.04$ breaths $/ \mathrm{min}$ at $\mathrm{T} 60$ in the $\mathrm{CHc}$ group $(\mathrm{P}=1)$. Continuous hypercapnia did not evoke significant long-lasting changes of the pPNA $60 \mathrm{~min}$ after the end of the stimulus compared to baseline $(+39.3 \pm 30.2 \%$ in the $\mathrm{CHc}$ group, $\mathrm{P}=1)$.
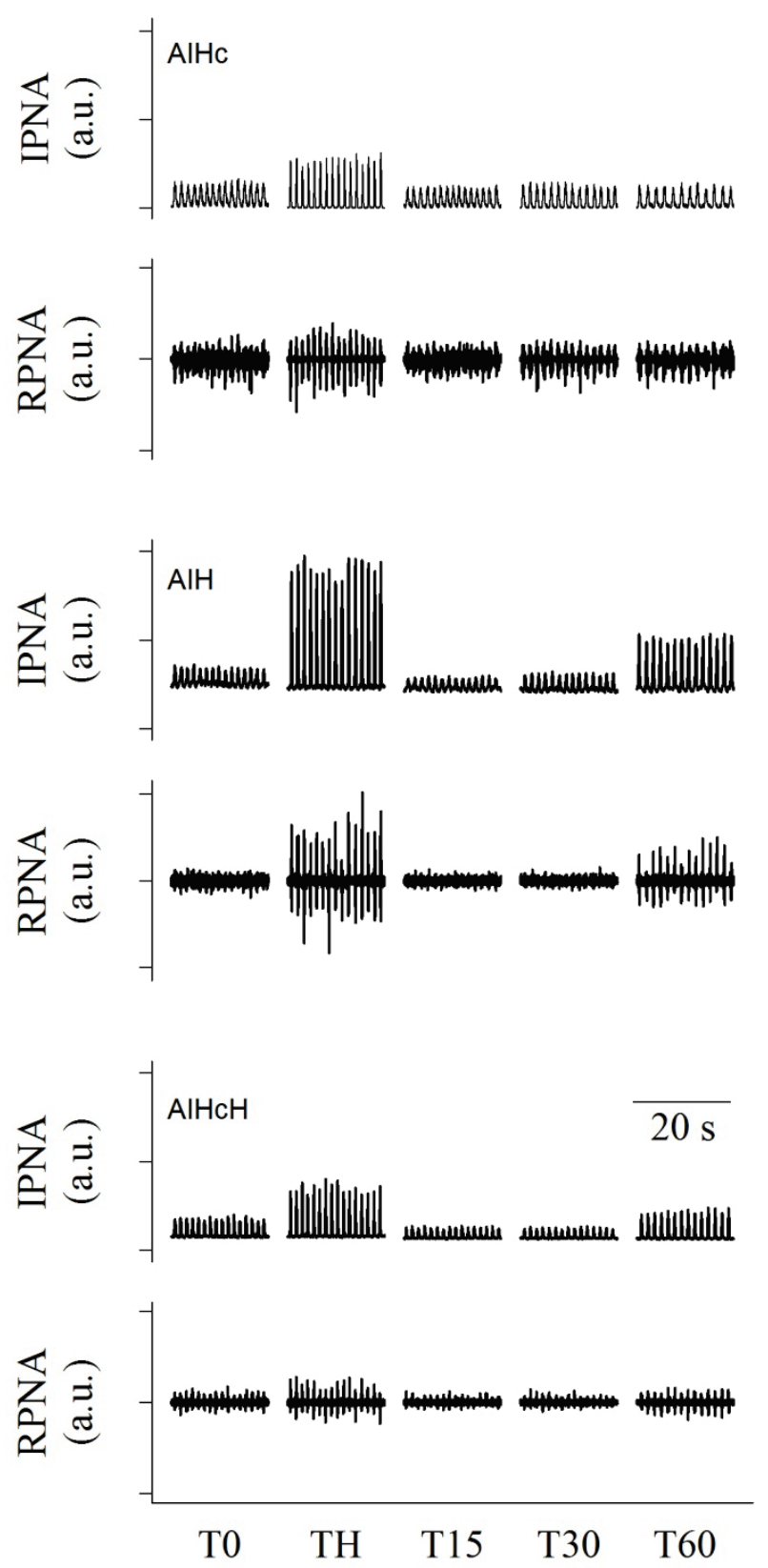

Fig. 1. Responses of integrated phrenic nerve activity (IPNA; arbitrary units, a.u.) and raw phrenic nerve activity (RPNA; arbitrary units, a.u.) following hypercapnic (AIHc), hypoxic (AIH), and combined hypercapnic and hypoxic stimuli $(\mathrm{AIHCH})$ in three rats. AIHc rat was exposed to acute intermittent hypercapnia, showing depression of the respiratory frequency at T60 (frequency pLTD). AIH rat was exposed to acute intermittent hypoxia, indicating pLTF at T60. Combined hypercapnia and hypoxia elicited non-significant increase in pPNA at T60. The phrenic nerve activity is shown during baseline conditions (T0), at the hypercapnic or hypoxic episode (TH), and 15 (T15), 30 (T30), and 60 (T60) min after the end of the last hypercapnic or hypoxic stimulus. 


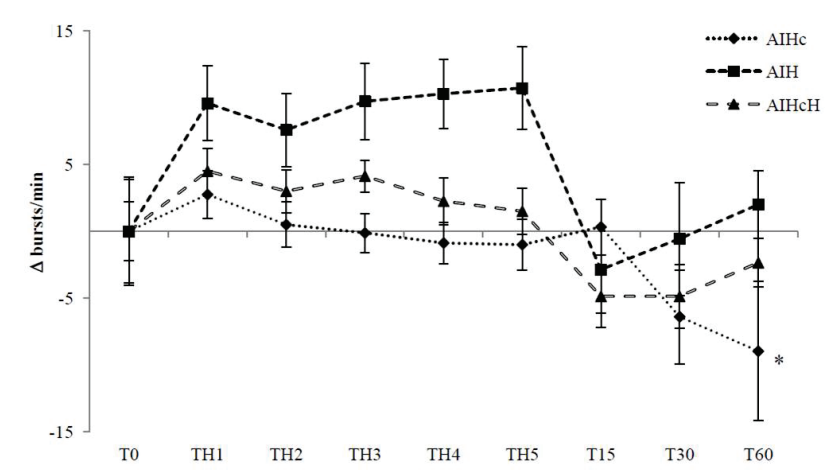

Fig. 2. Time course of changes in the phrenic burst frequency ( $\Delta$ bursts/min) in groups exposed to hypercapnic (AIHc), hypoxic stimulation (AIH), and combined hypercapnic and hypoxic stimulation $(\mathrm{AIHCH})$. Acute intermittent hypercapnia evoked frequency phrenic long-term depression (pLTD) 60 min after the end of the last hypercapnic stimulus. * Significantly different from baseline values, $\mathrm{P}<0.05$. T0 refers to baseline value before hypercapnia or hypoxia; TH1-5 represent five hypercapnic episodes, whereas T15, T30, and T60 are time points at 15, 30, and $60 \mathrm{~min}$ after the end of the hypercapnic or hypoxic stimulus.

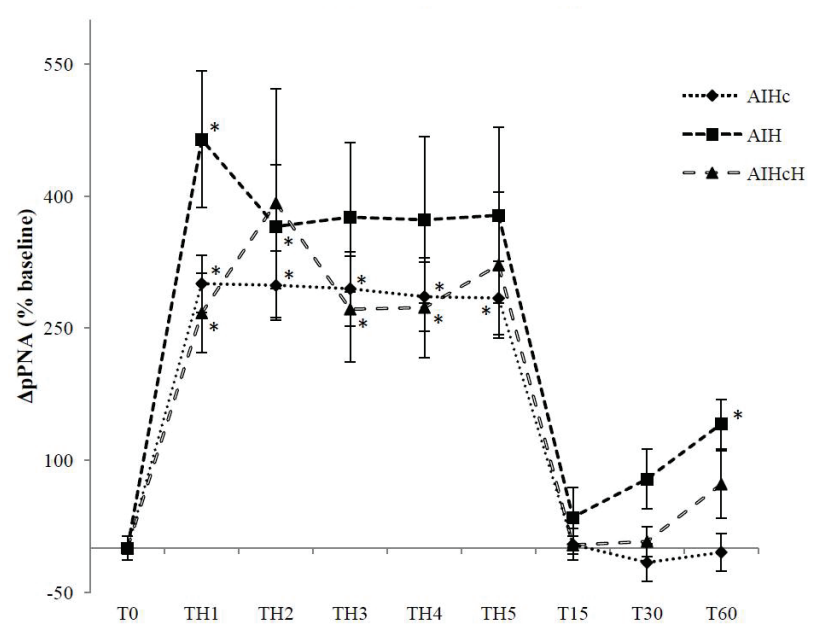

Fig. 3. Time course of changes in the peak phrenic nerve activity (pPNA) in groups exposed to hypercapnic stimulation (AIHc), hypoxic stimulation (AIH), and combined hypercapnic and hypoxic stimulation $(\mathrm{AIHCH})$. Exposure to AIH protocol evoked phrenic long-term facilitation (pLTF) at T60. * Significantly different from baseline values, $P<0.05$. T0 refers to baseline value before hypercapnia or hypoxia; TH1-5 represent five hypercapnic or hypoxic episodes, whereas T15, T30, and T60 are time points at 15,30 , and $60 \mathrm{~min}$ after the end of the hypercapnic or hypoxic stimulus.

\section{Effects of hypoxic stimuli}

Acute intermittent hypoxia did not evoke significant long-lasting changes of the respiratory frequency $60 \mathrm{~min}$ after the end of the stimuli, i.e. frequency was $37.71 \pm 3.87$ breaths $/ \mathrm{min}$ at $\mathrm{T} 0$ and
$39.71 \pm 2.55$ breaths $/$ min at $\mathrm{T} 60$ ( $\mathrm{P}=1$; Figs 1 and 2$)$. Still, acute intermittent hypoxia elicited a significant increase of pPNA at T60, i.e. pPNA increased by $141.0 \pm 28.2 \%$ compared to baseline ( $\mathrm{P}=0.015$; Figs 1 and 3), showing that pLTF developed.

Continuous hypoxia failed to evoke significant long-lasting changes of both, respiratory frequency $(43.71 \pm 2.36$ breaths $/ \mathrm{min}$ at $\mathrm{T} 0$ and $38.86 \pm 2.73$ breaths $/ \mathrm{min}$ at $\mathrm{T} 60, \mathrm{P}=1)$, and pPNA $(+22.8 \pm 47.4 \%$, $\mathrm{P}=1) 60 \mathrm{~min}$ after the end of the stimulus.

\section{Effects of combined hypoxic and hypercapnic stimuli}

Combined acute hypercapnia and hypoxia failed to evoke significant long-lasting changes of respiratory frequency $60 \mathrm{~min}$ after the end of the last stimuli. Respiratory frequency in $\mathrm{AIHcH}$ group was $40.5 \pm 2.2$ breaths $/ \mathrm{min}$ at $\mathrm{T} 0$ and $38.14 \pm 1.82$ breaths $/ \mathrm{min}$ at $\mathrm{T} 60$ ( $\mathrm{P}=1$; Figs 1 and 2). Combined hypoxic and hypercapnic stimulation failed to evoke significant change of the pPNA 60 min after the last stimulus compared to baseline values $(+72.6 \pm 38.4 \%, \mathrm{P}=1$; Figs 1 and 3$)$.

Regarding respiratory cycle parameters, there were no significant changes in $\mathrm{Ti}, \mathrm{Te}$, and $\mathrm{Ttot}$ at 15,30 , and $60 \mathrm{~min}$ after hypercapnic or hypoxic stimuli compared to baseline values in any group of the animals, as shown in Table 1. A significant change in respiratory rate should have also resulted in a significant change in Ttot and a lack thereof suggests that, because of the variability of the data, the study was underpowered to detect changes in respiratory timing.

Exposure to either intermittent (TH1-5) or continuous hypercapnic or hypoxic stimuli elicited an increase of pPNA in all groups of animals (Figs 1 and 3), but failed to significantly change respiratory frequency during the exposures (Figs 1 and 2).

There were no significant changes in $\mathrm{PaO}_{2}$, $\mathrm{PaCO}_{2}$, and $\mathrm{pH}$ values at $\mathrm{T} 0, \mathrm{~T} 15, \mathrm{~T} 30$, and $\mathrm{T} 60$ in any experimental group, as shown in Table 2.

Mean arterial pressure (MAP) tended to increase during the hypercapnic and combined hypercapnic/ hypoxic exposures and decreased during hypoxic exposures and then returned to baseline levels. Following exposures, MAP was successfully maintained relative to baseline in all experimental groups of animals until the end of the experiments. 
Table 1. Relative changes in $\mathrm{Ti}, \mathrm{Te}$, and Ttot during the protocol in experimental groups.

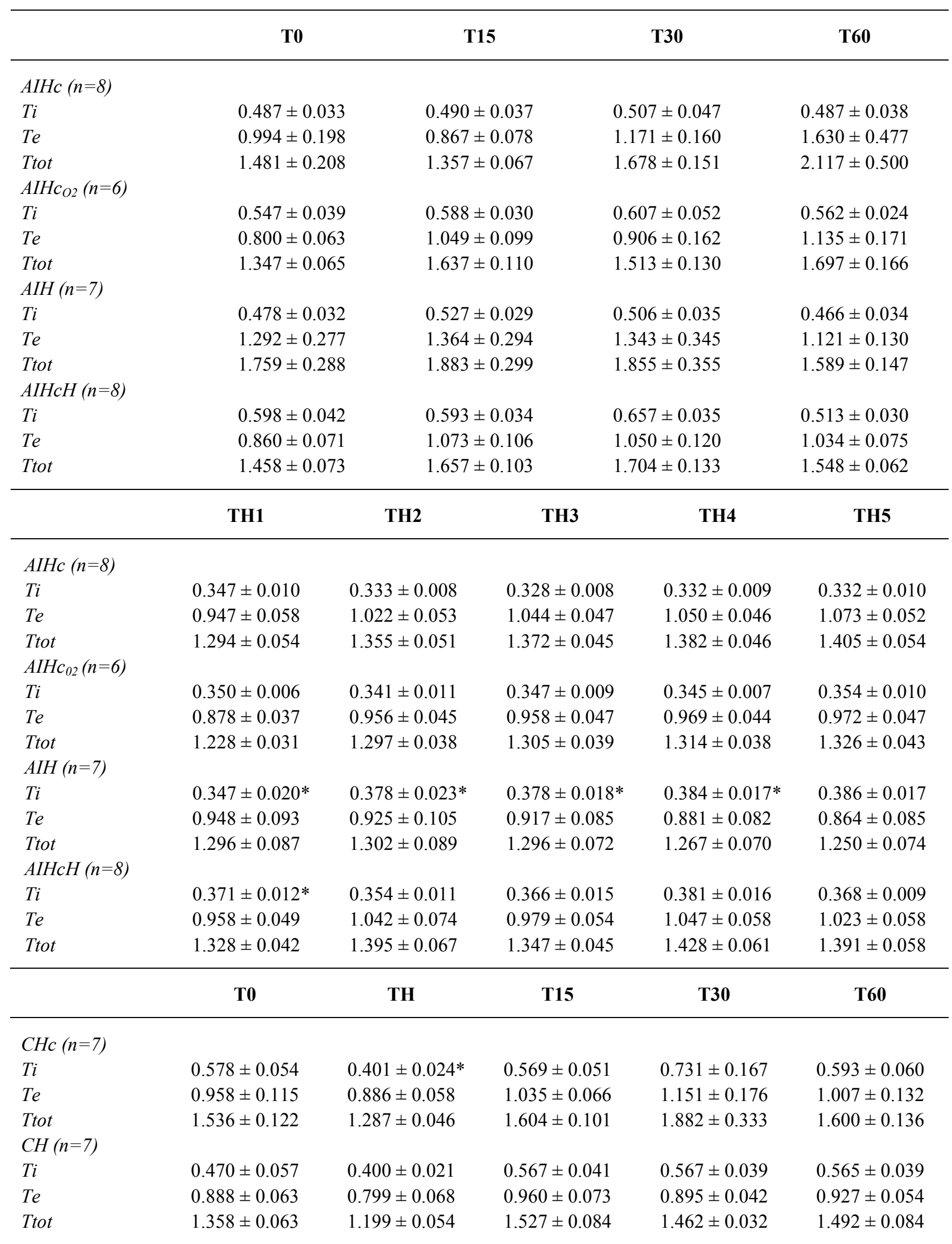

Data are expressed in seconds (mean \pm SEM). Ti, inspiratory time; Te, expiratory time; Ttot, total respiratory time; T0, baseline value before the first hypercapnia or hypoxia; $\mathrm{TH}$, continuous hypercapnia or hypoxia; $\mathrm{T} 15,15 \mathrm{~min}$ after the last hypercapnic or hypoxic episode; T30, 30 min after the last hypercapnic or hypoxic episode; T60, 60 min after the last hypercapnic or hypoxic episode; TH1-5, first-fifth hypercapnia or hypoxia. * Significantly different from baseline $(P<0.04)$. 
Table 2. Partial pressures of carbon dioxide $\left(\mathrm{PaCO}_{2}, \mathrm{~mm} \mathrm{Hg}\right)$, oxygen $\left(\mathrm{PaO}_{2}, \mathrm{~mm} \mathrm{Hg}\right)$, and $\mathrm{pH}$ in the arterial blood in all experimental groups of animals at different time points of experiment. There were no significant changes in $\mathrm{PaCO}_{2}, \mathrm{PaO}_{2}$, and $\mathrm{pH}$ values in comparison to baseline values.

\begin{tabular}{|c|c|c|c|c|c|c|}
\hline & \multicolumn{3}{|c|}{ T0 } & \multicolumn{3}{|c|}{ T15 } \\
\hline & $\mathrm{PaCO}_{2}$ & $\mathrm{PaO}_{2}$ & pH & $\mathrm{PaCO}_{2}$ & $\mathrm{PaO}_{2}$ & pH \\
\hline$A I H c(n=8)$ & $42.9 \pm 0.8$ & $73.2 \pm 6.0$ & $7.3 \pm 0.02$ & $47.1 \pm 2.3$ & $83.7 \pm 8.2$ & $7.3 \pm 0.03$ \\
\hline$A I H c_{O 2}(n=6)$ & $41.7 \pm 1.3$ & $258.9 \pm 11.7$ & $7.3 \pm 0.02$ & $44.5 \pm 2.0$ & $258.1 \pm 20.0$ & $7.3 \pm 0.03$ \\
\hline $\mathrm{CHc}(n=7)$ & $44.1 \pm 0.9$ & $80.7 \pm 5.9$ & $7.3 \pm 0.03$ & $44.1 \pm 1.6$ & $92.7 \pm 8.7$ & $7.3 \pm 0.03$ \\
\hline$A I H(n=7)$ & $42.0 \pm 0.5$ & $226.2 \pm 17.0$ & $7.3 \pm 0.03$ & $41.7 \pm 1.3$ & $251.2 \pm 10.0$ & $7.3 \pm 0.03$ \\
\hline $\mathrm{CH}(n=7)$ & $43.6 \pm 1.8$ & $254.7 \pm 5.1$ & $7.3 \pm 0.01$ & $41.8 \pm 1.6$ & $275.9 \pm 3.6$ & $7.2 \pm 0.02$ \\
\hline \multirow[t]{3}{*}{$A I H c H(n=8)$} & $44.7 \pm 0.7$ & $262.6 \pm 7.5$ & $7.3 \pm 0.01$ & $45.3 \pm 0.9$ & $270.9 \pm 7.1$ & $7.2 \pm 0.01$ \\
\hline & \multicolumn{3}{|c|}{ T30 } & \multicolumn{3}{|c|}{ T60 } \\
\hline & $\mathrm{PaCO}_{2}$ & $\mathrm{PaO}_{2}$ & pH & $\mathrm{PaCO}_{2}$ & $\mathrm{PaO}_{2}$ & $\mathrm{pH}$ \\
\hline $\operatorname{AIHC}(n=8)$ & $40.6 \pm 2.0$ & $89.2 \pm 8.7$ & $7.2 \pm 0.1$ & $42.6 \pm 1.6$ & $81.9 \pm 10.1$ & $7.3 \pm 0.02$ \\
\hline$A I H c_{O 2}(n=6)$ & $42.1 \pm 1.6$ & $278.0 \pm 8.7$ & $7.3 \pm 0.02$ & $40.4 \pm 1.1$ & $263.4 \pm 12.9$ & $7.3 \pm 0.04$ \\
\hline $\mathrm{CHc}(n=7)$ & $43.5 \pm 1.2$ & $92.0 \pm 8.5$ & $7.3 \pm 0.03$ & $41.8 \pm 1.2$ & $92.8 \pm 8.9$ & $7.3 \pm 0.03$ \\
\hline$A I H(n=7)$ & $40.3 \pm 1.3$ & $253.2 \pm 9.0$ & $7.2 \pm 0.03$ & $41.7 \pm 1.4$ & $238.5 \pm 13.5$ & $7.2 \pm 0.04$ \\
\hline $\mathrm{CH}(n=7)$ & $39.9 \pm 1.0$ & $270.0 \pm 3.6$ & $7.3 \pm 0.03$ & $40.3 \pm 1.3$ & $274.1 \pm 4.9$ & $7.2 \pm 0.04$ \\
\hline $\mathrm{AIHcH}(n=8)$ & $43.1 \pm 0.8$ & $276.4 \pm 6.6$ & $7.2 \pm 0.02$ & $43.9 \pm 0.9$ & $252.2 \pm 18.8$ & $7.2 \pm 0.02$ \\
\hline
\end{tabular}

Data are presented as mean \pm SEM. T0, baseline value before hypercapnia or hypoxia; T15, T30, and T60, 15, 30, and 60 min after the stimulus.

\section{Discussion}

The results of this study showed that acute intermittent hypercapnia evoked the development of frequency pLTD in anesthetized rats. In animals exposed to the acute intermittent hypoxia protocol, amplitude pLTF was elicited 60 min after the end of the last hypoxic stimulus, i.e. pLTF was induced. Combined intermittent hypercapnia and hypoxia as well as continuous hypercapnia or hypoxia failed to induce phrenic nerve plasticity $60 \mathrm{~min}$ after the end of the last hypercapnic/ hypoxic stimulus. Thus, it appears that intermittency of the hypercapnic or hypoxic stimulus is needed to evoke distinct forms of respiratory plasticity.

The majority of previous studies investigating mechanisms of hypercapnia induced respiratory plasticity used the model of urethane anesthetized rats exposed to three 5-min long hypercapnic stimulations separated by normocapnic recovery periods (Bach and Mitchell 1998, Baker et al. 2001). Although repeated intracarotid injections of $\mathrm{CO}_{2}$ saturated saline evoked pLTF, systemic exposure to intermittent hypercapnia resulted in pLTD (Morris et al. 1996, Bach and Mitchell 1998). Similarly, in our study, systemic exposure to the protocol of acute intermittent hypercapnia resulted in long lasting depression of the phrenic nerve frequency and had no long lasting effects on phrenic nerve amplitude. Systemic application of high levels of $\mathrm{CO}_{2}$ stimulates both central and peripheral chemoreceptors resulting in the facilitation of the phrenic nerve during exposure. Morris and colleagues (1996) injected $\mathrm{CO}_{2}$ saturated saline directly into the carotid artery and stimulated mainly carotid chemoreceptors, with minimal effects on the central nervous system (Morris et al. 1996). Thus, one might presume that the central effects of high levels of $\mathrm{CO}_{2}$ are crucial in the development of ventilatory depression. Moreover, it was suggested that hypercapnia induces an increase of extracellular $\mathrm{HCO}_{3}^{-}$, and as increased concentrations of $\mathrm{HCO}_{3}{ }^{-}$in the brain persist long after cessation of hypercapnia, it could be associated with long lasting depression of the phrenic nerve (Bach and Mitchell 1998). Still, in our study blood $\mathrm{pH}$ levels were maintained throughout the protocol and frequency pLTD developed late (60 min) after cessation of hypercapnic stimulation, so we do not assume that the elevated levels of brain $\mathrm{HCO}_{3}{ }^{-}$could be responsible for the observed frequency $\mathrm{pLTD}$. In addition, in our study continuous hypercapnia failed to evoke frequency pLTD. 
On the other hand, in our study both intermittent and continuous hypercapnia protocols failed to evoke amplitude pLTD. Cumulative effects of severe, continuous hypercapnia on the noradrenergic system were suggested to be the reason for the development of pLTD in some studies (Baker et al. 2001). However, continuous hypercapnia in our study failed to evoke pLTD. A possible explanation might be that the pattern sensitive signaling processes indicating that intermittent, but not continuous stimulus enhances gene expression important in long-lasting neuronal plasticity and longterm memory formation (Freudenthal and Romano 2000). In our study, the lack of apparent amplitude pLTD following intermittent hypercapnia might be the consequence of the simultaneous development of serotonin dependant $\mathrm{pLTF}$, which counterbalanced the noradrenalin dependant pLTD.

In the last two decades, pLTF evoked by acute intermittent hypoxia was in the research focus of many studies (Fuller et al. 2000, Dale-Nagle et al. 2010, Mitchell et al. 2001). It is known that pLTF depends on the serotonergic neuromodulation, as well as the specific pattern and timing of stimulus performance (Pavlinac et al. 2011, Pecotic et al. 2009, Valic et al. 2010, Mateika and Sandhu 2011). Results from our study confirm previous research regarding the importance of periodicity of hypoxic stimulation in the development of respiratory plasticity, since only acute intermittent, but not continuous hypoxia evoked pLTF.

Our results are in accordance with previous studies investigating LTF in anesthetized rats (Bach and Mitchell 1996, Pavlinac et al. 2011) which reported a decrease in blood pressure during hypoxic exposures. Although hypoxia results in strong sympathetic stimulation mediated by chemosensitive cells in carotid bodies (Zoccal et al. 2014), it seems that the hemodynamic response may depend on the level of hypoxia (Sabino et al. 2013). Indeed, hypoxia with 8, 10, or $15 \% \mathrm{O}_{2}$ produced slight hypotension independent of carotid chemoreceptors that might be explained by the release of adenosine acting on endothelial $A_{1}$ receptors to stimulate the synthesis and release of NO which induces vasodilatation and consequent hypotension (Ray and Marshall 2005).

We are aware that the mixture of $15 \% \mathrm{CO}_{2}$ in air would refer to decreased $\mathrm{PaO}_{2}$ in our protocol possibly leading to mild-hypoxic-hypercapnic exposure. However, gas mixture of $15 \% \mathrm{CO}_{2}$ in air would lead to the reduction of $\mathrm{O}_{2}$ in that mixture to about $17-18 \%$ which would represent only mild hypoxic stimulus that could not evoke long term changes of the phrenic nerve activity (Mateika and Sandhu 2011) and thus should not interfere with the final result. In addition, in order to investigate severe hypoxic $\left(9 \% \mathrm{O}_{2}\right)$ and severe hypercapnic stimulus $\left(15 \% \mathrm{CO}_{2}\right)$ we performed a separate set of experiments using combination of $9 \% \mathrm{O}_{2}$ and $15 \% \mathrm{CO}_{2}$ in nitrogen. Although hyperoxic hypercapnia used in the literature (Bach and Mitchell 1998) represents better choice to avoid confounding effect of hypoxic stimulus, we believe that hypoxic stimulus using $15 \% \mathrm{CO}_{2}$ in air was not sufficient to affect phrenic LTF or LTD.

The biological and clinical significance of intermittent hypoxia has been well recognized in recent years. It has been suggested that intermittent hypoxia might have a beneficial role in sleep related breathing disorders and could be a viable means of restoring respiratory functions in ventilatory control disorders (Mateika and Narwani 2009, Gaultier 2008). However, although intermittent hypercapnia results in altered respiratory control, its short and long-term effects on respiratory control remain poorly studied (Steggerda et al. 2010).

In this study, we did not determine neurotransmitters involved in respiratory plasticity, exact mechanisms, and the precise site of action in the development of pLTD and pLTF. However, different protocols gave us insights into the importance of stimulus periodicity in the induction of distinct forms of respiratory plasticity. Some of the previous studies were conducted in background hyperoxia $\left(50 \% \mathrm{O}_{2}\right)$ to avoid additional hypoxic stimulus between challenging episodes (Bach and Mitchell 1998). However, high background levels of $\mathrm{O}_{2}$ might affect expression of pLTF and pLTD. In our study, some protocols were conducted in background normoxia (air, $21 \% \mathrm{O}_{2}$ ) because high levels of inspired $\mathrm{O}_{2}$ inhibit the action of $\mathrm{CO}_{2}$ and the peripheral chemoreceptors (Fitzgerald and Parks 1971).

Bearing in mind that intermittent hypoxia might have an important beneficial role in many pathological conditions (Dale et al. 2014), one might speculate about the physiological significance of hypercapnia induced respiratory plasticity. In our study hypercapnic exposures were severe $(15 \%)$ which is not likely to be seen in clinical practice, even in severe lung diseases or sleep disordered breathing. We are aware of the fact that at this level $\mathrm{CO}_{2}$ might have actions that are not related only to chemoreception. However, we believe that mechanistic insights into hypercapnia and combined hypercapnia and 
hypoxia induced respiratory plasticity might be useful in understanding respiratory plasticity and its role in different pathological conditions.

Previous studies showed that pLTD could be evoked following intermittent hypercapnic stimulus. The possible mechanisms of pLTD were proposed, indicating that hypercapnia-induced release of norepinephrine could act on $\alpha_{2}$-adrenergic receptors located on raphe serotonergic neurons to inhibit raphe excitability and release of serotonin. In our study, pLTD could be evoked if acute intermittent hypercapnic stimulus was performed, but not if concomitant hypercapnic and hypoxic stimuli were applied. Our results, in accordance with numerous previous studies confirmed that acute intermittent hypoxia induces the development of pLTF. However, in our study if acute intermittent hypoxia was combined with acute intermittent hypercapnia, pLTF was attenuated. It is well known that carbon dioxide levels might alter LTF expression in animals (Bach and Mitchell 1996, Fregosi and Mitchell 1994, Harris et al. 2006). It is possible that hypercapnia-induced activation of noradrenergic neurons in our study inhibited raphe serotonergic neurons and consequently led to attenuation of pLTF.

The impact of intermittent versus sustained hypercapnia and/or hypoxia on phrenic nerve plasticity was investigated and reported in numerous studies often using different species, substrains and protocols. In this study we present evidence about the pattern sensitivity of phrenic nerve plasticity in respect to different respiratory challenges compared in a single study on the same substrain of Sprague-Dawley rats. The results of this study indicate that acute intermittent hypercapnia elicits frequency pLTD, whereas acute intermittent hypoxia elicits amplitude pLTF in anesthetized rats. Combined hypercapnia and hypoxia, as well as continuous hypercapnic or hypoxic stimulus failed to evoke phrenic nerve plasticity. Thus, we conclude that intermittency of the hypercapnic or hypoxic stimulus is needed to evoke distinct forms of phrenic nerve plasticity.

\section{Conflict of Interest}

There is no conflict of interest.

\section{Acknowledgements}

The authors wish to thank Mrs. Jelena Baricevic for her technical assistance and Shelly Pranic, M.S., University of Split, School of Medicine for the language editing of the manuscript. This study was supported by the Croatian Science Foundation grant $09 / 165$ granted to M. Valic, and previous projects and equipment granted to Z. Dogas.

\section{References}

BACH KB, MITCHELL GS: Hypoxia-induced long-term facilitation of respiratory activity is serotonin dependent. Respir Physiol 104: 251-260, 1996.

BACH KB, MITCHELL GS: Hypercapnia-induced long-term depression of respiratory activity requires alpha2adrenergic receptors. J Appl Physiol 84: 2099-2105, 1998.

BAKER TL, MITCHELL GS: Episodic but not continuous hypoxia elicits long-term facilitation of phrenic motor output in rats. $J$ Physiol 529: 215-219, 2000.

BAKER TL, FULLER DD, ZABKA AG, MITCHELL GS: Respiratory plasticity: differential actions of continuous and episodic hypoxia and hypercapnia. Respir Physiol 129: 25-35, 2001.

BAKER-HERMAN TL, MITCHELL GS: Phrenic long-term facilitation requires spinal serotonin receptor activation and protein synthesis. $J$ Neurosci 22: 6239-6246, 2002.

BAKER-HERMAN TL, MITCHELL GS: Determinants of frequency long-term facilitation following acute intermittent hypoxia in vagotomized rats. Respir Physiol Neurobiol 162: 8-17, 2008.

CAREV M, VALIC M, PECOTIC R, KARANOVIC N, VALIC Z, PAVLINAC I, DOGAS Z: Propofol abolished the phrenic long-term facilitation in rats. Respir Physiol Neurobiol 170: 83-90, 2010.

DALE EA, BEN MABROUK F, MITCHELL GS: Unexpected benefits of intermittent hypoxia: enhanced respiratory and nonrespiratory motor function. Physiology (Bethesda) 29: 39-48, 2014.

DALE-NAGLE EA, HOFFMAN MS, MACFARLANE PM, MITCHELL GS: Multiple pathways to long-lasting phrenic motor facilitation. Adv Exp Med Biol 669: 225-230, 2010.

DEVINNEY MJ, HUXTABLE AG, NICHOLS NL, MITCHELL GS: Hypoxia-induced phrenic long-term facilitation: emergent properties. Ann N Y Acad Sci 1279: 143-153, 2013. 
DICK TE, HSIEH YH, MORRISON S, COLES SK, PRABHAKAR N: Entrainment pattern between sympathetic and phrenic nerve activities in the Sprague-Dawley rat: hypoxia-evoked sympathetic activity during expiration. $\mathrm{Am}$ J Physiol Regul Integr Comp Physiol 286: R1121-R1128, 2004.

DICK TE, HSIEH YH, WANG N, PRABHAKAR N: Acute intermittent hypoxia increases both phrenic and sympathetic nerve activities in the rat. Exp Physiol 92: 87-97, 2007.

FELDMAN JL, MITCHELL GS, NATTIE EE: Breathing: rhythmicity, plasticity, chemosensitivity. Annu Rev Neurosci 26: 239-266, 2003.

FITZGERALD RS, PARKS DC: Effect of hypoxia on carotid chemoreceptor response to carbon dioxide in cats. Respir Physiol 12: 218-229, 1971.

FREGOSI RF, MITCHELL GS: Long-term facilitation of inspiratory intercostal nerve activity following carotid sinus nerve stimulation in cats. J Physiol 477: 469-479, 1994.

FREUDENTHAL R, ROMANO A: Participation of Rel/NF-kappaB transcription factors in long-term memory in the crab Chasmagnathus. Brain Res 855: 274-281, 2000.

FULLER DD, BACH KB, BAKER TL, KINKEAD R, MITCHELL GS: Long term facilitation of phrenic motor output. Respir Physiol 121: 135-146, 2000.

GAULTIER C: Genetic Basis for Respiratory Control Disorders. Springer Science \& Business Media, 2007.

HARRIS DP, BALASUBRAMANIAM A, BADR MS, MATEIKA JH: Long-term facilitation of ventilation and genioglossus muscle activity is evident in the presence of elevated levels of carbon dioxide in awake humans. Am J Physiol Regul Integr Comp Physiol 291: R1111-R1119, 2006.

IVANCEV B, CAREV M, PECOTIC R, VALIC M, PAVLINAC DODIG I, KARANOVIC N, DOGAS Z: Remifentanil reversibly abolished phrenic long term facilitation in rats subjected to acute intermittent hypoxia. J Physiol Pharmacol 64: 485-492, 2013.

KINKEAD R, BACH KB, JOHNSON SM, HODGEMAN BA, MITCHELL GS: Plasticity in respiratory motor control: intermittent hypoxia and hypercapnia activate opposing serotonergic and noradrenergic modulatory systems. Comp Biochem Physiol A Mol Integr Physiol 130: 207-218, 2001.

MAHAMED S, MITCHELL GS: Simulated apnoeas induce serotonin-dependent respiratory long-term facilitation in rats. J Physiol 586: 2171-2181, 2008.

MARINOV V, VALIC M, PECOTIC R, KARANOVIC N, PAVLINAC DODIG I, CAREV M, VALIC Z, DOGAS Z: Sevoflurane and isoflurane monoanesthesia abolished the phrenic long-term facilitation in rats. Respir Physiol Neurobiol 189: 607-613, 2013.

MATEIKA JH, NARWANI G: Intermittent hypoxia and respiratory plasticity in humans and other animals: does exposure to intermittent hypoxia promote or mitigate sleep apnoea? Exp Physiol 94: 279-296, 2009.

MATEIKA JH, SANDHU KS: Experimental protocols and preparations to study respiratory long term facilitation. Respir Physiol Neurobiol 176: 1-11, 2011.

MITCHELL GS, BAKER TL, NANDA SA, FULLER DD, ZABKA AG, HODGEMAN BA, BAVIS RW, MACK KJ, OLSON EB JR: Invited review: Intermittent hypoxia and respiratory plasticity. J Appl Physiol 90: 2466-2475, 2001.

MORRIS KF, ARATA A, SHANNON R, LINDSEY BG: Long-term facilitation of phrenic nerve activity in cats: responses and short time scale correlations of medullary neurones. J Physiol 490: 463-480, 1996.

PAVLINAC I, PECOTIC R, DOGAS Z, VALIC M: Role of 5-HT(1A) receptors in induction and preservation of phrenic long-term facilitation in rats. Respir Physiol Neurobiol 175: 146-152, 2011.

PAVLINAC DODIG I, PECOTIC R, VALIC M, DOGAS Z: Acute intermittent hypoxia induces phrenic long-term facilitation which is modulated by 5 -HT1A receptor in the caudal raphe region of the rat. J Sleep Res 21 : 195-203, 2012.

PECOTIC R, DOGAS Z, VALIC Z, VALIC M: Blockade of 5-HT(1A) receptors in the phrenic nucleus of the rat attenuated raphe induced activation of the phrenic nerve activity. J Physiol Pharmacol 60: 167-172, 2009.

RAY CJ, MARSHALL JM: Measurement of nitric oxide release evoked by systemic hypoxia and adenosine from rat skeletal muscle in vivo. J Physiol 568: 967-978, 2005.

RYAN S, NOLAN P: Long-term facilitation of upper airway muscle activity induced by episodic upper airway negative pressure and hypoxia in spontaneously breathing anaesthetized rats. J Physiol 587: 3343-3353, 2009. 
SABINO JP, OLIVEIRA MD, GIUSTI H, GLASS ML, SALGADO HC, FAZAN R JR: Hemodynamic and ventilatory response to different levels of hypoxia and hypercapnia in carotid body-denervated rats. Clinics (Sao Paulo) 68: 395-399, 2013.

STEGGERDA JA, MAYER CA, MARTIN RJ, WILSON CG: Effect of intermittent hypercapnia on respiratory control in rat pups. Neonatology 97: 117-123, 2010.

VALIC M, PECOTIC R, PAVLINAC I, VALIC Z, PEROS K, DOGAS Z: Microinjection of methysergide into the raphe nucleus attenuated phrenic long-term facilitation in rats. Exp Brain Res 202: 583-589, 2010.

ZOCCAL DB, FURUYA WI, BASSI M, COLOMBARI DS, COLOMBARI E: The nucleus of the solitary tract and the coordination of respiratory and sympathetic activities. Front Physiol 5: 238, 2014. 